\title{
Construir currículum desde abajo: avanzando en la documentación de una propuesta curricular en el contexto del COVID-19
}

\author{
CONSTANZA CÁRDENAS ALARCÓN ${ }^{*}$ \\ Universidad de Santiago de Chile - Chile \\ SEBASTIÁN GUERRERO LACOSTE* \\ Universidad Metropolitana de Ciencias de la Educación - Chile
}

DANIEL JOHNSON MARDONES***

Universidad de Chile - Chile

Recibido el 26-01-21; evaluado el 11-02-20;

aceptado el 26-02-21

\section{Resumen}

Los cambios a que se vieron enfrentados los sistemas escolares, y las sociedades en general, a causa del COVID-19 llevaron a estos de una excepcionalidad de la pandemia a la situación de emergencia como regla general. El presente trabajo describe una experiencia de construcción curricular desarrollada por los y las trabajadoras de la educación del primer ciclo de primaria en una escuela chilena. El proceso de construcción curricular participativa se basa en la propuesta de los temas generadores de Paulo Freire, donde los procesos de construcción del

\footnotetext{
* Docente en la Universidad de Santiago de Chile (USACH). Magíster (c) en Educación mención Currículum y Comunidad Educativa, Universidad de Chile. Colaboradora del Núcleo de Estudio Currículum, Conocimiento y Experiencia Escolar. Correo electrónico: constanzacardenas@ug.uchile.cl. https://orcid.org/0000-0002-6267-5404

** Profesor de Educación Física, Deportes y Recreación de la UMCE (Chile). Magíster (c) en Educación mención Currículum y Comunidad Educativa, Universidad de Chile. Colaborador del Núcleo de Estudio Currículum, Conocimiento y Experiencia Escolar. Investigador integrante del Grupo de Trabajo CLACSO «Educación Popular y Pedagogías Críticas». Educador Popular de la Escuela Pública Comunitaria y Profesor de Educación Física en el Colegio San José de Peñalolén. Correo electrónico: sebastianguerrero@ug.uchile.cl. https://orcid.org/0000-0002-4311-4388

*** Profesor de Historia y Geografía por la Universidad de Concepción. Magíster en Curriculum y Comunidad Educativa por la Universidad de Chile. Ph. D. en Curriculum e Instrucción por la Universidad de Illinois at Urbana Champaign. Profesor asociado del Departamento de Educación de la Facultad de Ciencias Sociales de la Universidad de Chile. Coordinador del Núcleo de estudio Curriculum, Conocimiento y Experiencia Escolar. Correo electrónico: djohnson@uchile.cl. https:// orcid.org/0000-0002-1023-8915
} 
contenido de los programas de estudio se basan en un tema y/o un problema de relevancia para la comunidad educativa y a los intereses de los y las estudiantes.

Palabras clave: currículum, educación en pandemia, construcción curricular participativa.

\section{Building curriculum from the bottom: documenting a curriculum proposal in the context of COVID-19}

\section{Abstract}

The changes that school systems and societies due to COVID-19 were faced moving from an exceptionality of emergency to a situation where the emergency become the general rule. The present work describes an experience of curriculum construction developed by the education workers of the first cycle of primary school in a Chilean school. The process of participatory curriculum construction was based on the proposal of the generating themes of Paulo Freire, where the processes of construction of the content of the study programs are based on a theme and / or a problem of relevance to the educational community and to the interests of the students.

Keywords: Curriculum, pandemic education, participatory curriculum construction.

\section{Construindo currículo a partir de baixo: avançando na documentaçáo de uma proposta curricular no contexto do COVID-19}

\section{Resumo}

As mudanças que os sistemas escolares e as sociedades em geral enfrentaram devido ao COVID-19 levaram-nos da excepcionalidade da emergência a uma situação de emergência como regra geral. O presente trabalho descreve uma experiência de construção curricular desenvolvida por trabalhadores da educação do primeiro ciclo do ensino fundamental de uma escola chilena. $\mathrm{O}$ processo de construção curricular participativa é baseado na proposição dos temas geradores de Paulo Freire, onde os processos de construçáo dos conteúdos dos programas de estudos são baseados em um tema e / ou problema de relevância para a comunidade educacional e para o interesses dos alunos.

Palavras-chave: currículo, educação pandêmica, construção curricular participativa. 


\section{INTRODUCCIÓN}

Los cambios a que se vieron enfrentados los sistemas escolares, y las sociedades en general, a causa del COVID-19 llevaron a estos de una excepcionalidad de la emergencia a la situación de emergencia como regla general. En efecto, la educación en emergencia había estado asociada desde ya hace unas décadas a procesos de desplazamiento de población, catástrofes naturales, y guerra. Ya en 2015, afirmaba un informe de Unesco (2017) titulado Educación en emergencias y crisis que, «A[a]sistimos a la mayor crisis humanitaria desde la II Guerra Mundial», haciendo referencia a la situación de 60 millones de desplazados por causa de conflictos armados, desastres naturales o proyectos de infraestructura. El informe constataba, además, que los desastres de la sociedad amplían las brechas de desigualdad. A pesar de la asertividad de estas afirmaciones, la situación desatada por el COVID-19 llevó esta situación a un nivel planetario, donde también se visualizan las enormes brechas de desigualdad en las que viven diariamente las poblaciones a nivel mundial y al interior de cada país.

La generalidad y prolongación de la emergencia, la permanencia de la crisis, la continuidad de la excepcionalidad y la mantención de los estados de excepción decretados para hacer frente a la misma, en lo que respecta a los sistemas escolares, marcaron una suspensión del tiempo y los espacios escolares a la vez que un esfuerzo por mantenerlos. En este sentido, Díaz-Barriga (2020) ha observado que «estamos ante un hecho inédito: la pérdida del espacio escolar y del aula» (p. 20). Y continúa este autor adelantando, que

Q[q]uizá el cumplimiento, en cierta forma, de la profecía que habían prefigurado en los años setenta del siglo pasado Illich, con su libro La sociedad desescolarizada, y Reimers, con La escuela ha muerto. El aislamiento social nos acerca de alguna forma a esta situación. (p. 20)

La referencia de Díaz-Barriga a la crítica radical de la escolaridad moderna de la década de 1960 resuena con la crítica actual a la nueva ola de estandarización instrumental a nivel global, proceso que también resuena con la ola tecnicista de esa década que constituyó el contexto en el cual la referida crítica radical a la escuela moderna fue formulada. En ese sentido, conviene preguntarse si la pandemia actual es o ha estado siendo una oportunidad, un paréntesis, o un lugar-tiempo, donde a lo menos se relativiza lo que Agamben (2003) llama la "expropiación de la experiencia», y viene a legitimar también el saber de la experiencia de los educadores y comunidades que se han visto en la necesidad de tomar decisiones más allá de la normatividad centralizada de los sistemas escolares 
¿Es entonces posible pensar en la educación en tiempos de pandemia, también, como una oportunidad, un paréntesis, un tiempo-lugar, una posibilidad de experimentar una educación más allá de la escolaridad moderna, y por lo mismo colonial? ¿¿Se trata de una posibilidad de desescolarización de la educación o una expansión de la escolaridad al espacio social más íntimo de nuestro cotidiano? La respuesta es probablemente relativa.

En este texto, asumiendo el cuestionamiento anterior, se documenta una experiencia en que la posibilidad fue posible y la oportunidad se tomó construyendo un curriculum participativamente en un establecimiento educacional de Santiago de Chile. Así, el presente trabajo describe un proceso de construcción y desarrollo curricular llevado a cabo por los y las trabajadoras de la educación del primer ciclo de primaria en una escuela chilena. El proceso de construcción curricular participativa se basa en la propuesta de los temas generadores de Paulo Freire (1970, 2008), donde los procesos de construcción del contenido de los programas de estudio se centran en un tema y/o un problema de relevancia para la comunidad educativa, y en los intereses de los y las estudiantes. La experiencia se realiza en el marco de la crisis sanitaria del COVID-19 y corresponde a una propuesta de educación remota.

\section{MarCo teórico}

\subsection{Contexto}

En las últimas décadas, el sistema escolar chileno ha sido objeto de grandes cambios y críticas, y es que en diversas instancias ha sido profundamente cuestionado por diversos actores, como las movilizaciones estudiantiles de 2001, 2006 y 2011; del profesorado en 2015 y 2018; desde la opinión pública y parte de la academia; e incluso desde organismos internacionales como la OCDE (2004). Las movilizaciones estudiantiles impulsaron los cambios más profundos desde el inicio de la transición a la democracia (Assael, Cornejo, Albornoz, Echeberriegaray, Ligueño y Palacios, 2015), abriendo un ciclo de impugnación y visibilización de la crisis social que ha generado un sistema escolar regido mediante lógicas empresariales, por las leyes del mercado, y altamente privatizado; por otro lado y ante esta situación, se ha venido reivindicando la necesidad de garantizar el derecho a la educación pública, que en Chile no se encuentra garantizado.

Desde el Estado de Chile, se han establecido varios compromisos a nivel nacional e internacional para garantizar una educación democrática, equitativa e inclusiva, acuerdos que no se han llevado a la práctica independiente 
de su importancia a nivel internacional, como es el caso de la Declaración de Incheon el ańo 2015. Ha sido ampliamente documentado que el sistema escolar chileno actual se ha conformado desde una perspectiva profundamente neoliberal, la cual ha sido fuertemente cuestionada por múltiples razones, de las cuales nos parece relevante mencionar dos. La primera, en la Constitución de 1980, el Estado consignó el principio de subsidiariedad en la prestación de servicios educativos, como consecuencia de esto se ha generado una alta privatización, competencia y lucro en el sistema escolar (Cornejo, 2018). La segunda tiene relación con que otros derechos sociales también fueron negados en esta Constitución y en las políticas posteriores, todo esto en un momento donde los grandes grupos económicos del país experimentaban un período de gran enriquecimiento (Sánchez y Sobarzo, 2010) lo que ha provocado una creciente y abismante segregación y desigualdad vivida por la población. Sin duda, estos problemas de tipo estructural, la creciente deslegitimación del sistema político por casos de corrupción y por la implementación de un sistema democrático abiertamente no participativo (Grez y Foro por la Asamblea Constituyente, 2015), y la emergencia e impugnación de diversos movimientos sociales durante dos décadas, han sido elementos de base para el estallido social y la revuelta popular iniciada en octubre de 2019, a partir de la cual se inicia un nuevo ciclo con el cambio de la constitución de la dictadura.

Estos dos elementos, a saber, la inexistencia de la garantía del derecho a la educación pública y la desigualdad social, son grandes dificultades que impiden la construcción de una educación pública, democrática e inclusiva. En los planteamientos de Pablo Gentili (2001), a nivel social, en Chile se ha formado un apartheid educativo mediante las políticas neoliberales, donde el sistema escolar produce y reproduce las diferencias entre clases sociales a través de variados mecanismos de exclusión de los grupos oprimidos. Incluso un organismo como la OCDE (2004), señaló en el informe de Revisión de Politicas Nacionales de Educación: Chile, que "el sistema educacional está conscientemente estructurado por clases [... [ [y que] la democracia no se ve favorecida por una estratificación tan intensa»(OCDE, 2004, p. 277).

La abismante desigualdad socioeconómica y el establecimiento de una democracia de baja intensidad con un carácter autoritario tienen sus efectos, y se hacen carne al interior de las comunidades educativas, sobre todo de aquellas que se encuentran en contextos de mayor exclusión y vulneración de derechos. La segregación y la negación de derechos se refleja en las escuelas, donde los indicadores educativos de calidad apuntan a una correlación entre los logros de aprendizaje del estudiantado y el nivel socioeconómico de su familia (Foro por el Derecho a la Educación Pública, 2019). 
Es importante destacar que, a nivel internacional, la garantía del derecho a la educación no se restringe al acceso (tener la posibilidad de acceder al sistema educativo en sus distintos niveles), sino también a criterios relacionados a una educación democrática como la asequibilidad (donde se respete la diversidad cultural y la libertad), la adaptabilidad (donde las instituciones estén enmarcadas en los derechos humanos y se adapten a sus estudiantes y comunidades educativas), y la aceptabilidad (tener una experiencia educativa aceptable y digna, considerando en los procesos educativos las vidas y los derechos de los niños, niñas y jóvenes) (Tomasevski, 2004). Entendido así, el derecho a la educación pública no se garantiza posibilitando la asistencia de estudiantes a una escuela, sino que involucra una serie de elementos como condiciones dignas, respeto de la diversidad, consideración y adaptación a las experiencias de vida de estudiantes y comunidades educativas, participación activa del proceso de los diversos actores, entre otros. A modo de síntesis, entre las múltiples causas que han provocado la crisis política y el inicio de un proceso constituyente, estarían la creciente desigualdad, la negación de derechos en la Constitución de 1980 y la crisis de legitimidad del sistema político. Estos elementos se han desarrollado a la par con la crisis que ha experimentado la educación chilena en el último tiempo, producto de la implementación de políticas neoliberales que han profundizado la segregación desde el ámbito educativo. Esto ha sido posible, en gran parte, debido a la ausencia de garantía del derecho a la educación pública en la Constitución de la dictadura, garantía que no solo se logra mediante el acceso y la permanencia en el sistema escolar, sino también por criterios de pertinencia y de democratización referidos a la asequibilidad, la adaptabilidad y a la aceptabilidad de la educación.

En lo que respecta a la construcción curricular, los procesos de diseño y desarrollo; esto es, la forma en que se diseña la prescripción curricular nacional y los distintos procesos que ocurren hasta su puesta en práctica por los profesionales de la educación a nivel de la escuela y el aula. En general, las teorías curriculares orientadas a los procesos (Glatthorn, Boschee, Whitehead y Boschee, 2019) se han preocupado de ofrecer sistemas conceptuales que ayuden a guiar el proceso de diseño y desarrollo curricular, y de pensar el currículum como sistema (Null, 2011). Con todo, la preocupación por la práctica curricular y la relación currículum -profesorado ha proveído una importante literatura que pone el acento en la descripción y análisis de dichos procesos con mayor énfasis en los procesos de negociación y resignificación de las definiciones curriculares. En Chile esta labor está marcada por una separación de los procesos de diseño y desarrollo curricular; que, aunque incorpora un cierto espacio para la toma de decisiones por parte de los actores locales, las 
posibilidades terminan siendo limitadas y restringidas dada la influencia que ejercen las estructuras curriculares en el desarrollo curricular, particularmente el currículum evaluado.

Un concepto importante en la relación entre los procesos de diseńo y desarrollo curricular, y por tanto en la construcción curricular participativa, es el de currículum enseñado. Este concepto se desarrolla en el seno de las teorías curriculares orientadas al proceso (Glatthorn et al., 2019). El currículum enseńado es el currículum tal como es entendido y puesto en práctica por los profesores (Caro y Aguilar, 2018; Espinoza Guzmán y Riquelme, 2018; Ferrada, 2012, 2001; Guzmán, 2001; Lozano, 2018; Mesa, Pascual y Pinto, 2006; Priestley, Edwards, Priestley y Miller, 2012; Rivera, 2009). Este es el currículum realmente puesto en práctica por los profesores, el currículum reinventado en la enseñanza, el currículum que «salta del papel y toma vida en el currículum de la escuela» (Hlebowitsh, 2009, p. 22). Este «salto» difiere en su naturaleza y la forma en que se da, pero la distancia entre lo que ha sido puesto en "papel» y aquello que se hace "vida» en las aulas es un punto crítico, tanto para el deseo de una implementación efectiva por parte de los responsables del sistema escolar, cuanto para el desarrollo de la autonomía profesional de los profesores. En este sentido, cuando consideramos los procesos de diseńo y desarrollo curricular desde el punto de vista de la relación currículum-profesorado nos encontramos con la tensión entre las perspectivas tecnificadoras que han dominado secularmente estos procesos y la producción académica que criticó la racionalidad tyleriana desde fines de 1960, y que desarrolló las concepciones sobre los profesores como investigadores (Stenhouse, 1987), como intelectuales (Giroux, 1990), como planificadores curriculares (Connelly y Clandinin, 1988), como hacedores o constructores de currículum (Clandinin y Connelly, 1992), como profesionales reflexivos (Schön, 1982; Imbernón, 1996; Wesbury, 2000), entre otras.

En el contexto chileno, Silva $(2001,2002,2017)$ ha argumentado que, como cultura normativa, las prescripciones curriculares se conectan y dialogan dinámicamente con las culturas organizacionales y profesionales. En este proceso, los profesores toman decisiones con ciertos criterios pedagógicos (Gysling, 1992). Estudiando el proceso de planificación en las escuelas primarias y secundarias, Erazo (2011) ha señalado que la planificación curricular puede ser una estrategia de mejora del currículum oficial. Sin embargo, la planificación es solo un aspecto de la toma de decisiones de los profesores. En este sentido, Guzmán (2010) ha estudiado el concepto de interacciones emergentes para explicar la "construcción cotidiana del currículo» (p. 319). Así, los profesores no serían solo el adaptador de las prescripciones curriculares, 
sino también creadores curriculares. En el mismo sentido, utilizando una perspectiva crítico-comunicativa, Ferrada (2001) utiliza el concepto de «entender las comunidades» para estudiar cómo los profesores utilizan lo que ella llama un modelo de diálogo-aprendizaje. Según esta autora, este enfoque puede conducir a mejoras significativas en el aprendizaje de los estudiantes, especialmente para aquellos y aquellas que viven en situaciones de exclusión.

\subsection{Pensando currículum en tiempos de pandemia}

Al hablar de currículum, es importante señalar la concepción que tengamos de este en tanto hacemos visible el interés que lo orienta y el proyecto de sociedad que se busca conformar mediante las experiencias educativas. Para esto, Shirley Grundy (1987), basándose en la teoría de los intereses cognitivos fundamentales de Jürgen Habermas, proporciona un marco con el cual es posible observar diferentes prácticas escolares y atribuirles un sentido al proponer tres clasificaciones de currículum según el interés predominante en ellos. Esto es: el currículum técnico, asociado al interés por el control; el currículum práctico, referido al interés de la comprensión; y el currículum crítico, orientado desde el interés por la emancipación social.

A partir de esta clasificación, Ferrada (2001) y Pinto (2008) han señalado que la concepción hegemónica del currículum en Chile desde 1960 a la fecha correspondería a un interés y una racionalidad técnico-instrumental, ya que se enfoca en el currículum como un producto escrito, en el estudiante como un producto del proceso de enseńanza, en el control de los aprendizajes y la estandarización, en la transmisión de conocimientos y/o competencias descontextualizadas y ahistóricas, y con una insalvable separación entre quienes detentan el rol de «expertos» que diseñan el currículum, y el profesorado que solo lo implementa, aplica o desarrolla. Es importante señalar también que, a pesar de que la gestión del sistema escolar chileno se ha descentralizado, la toma de decisiones curriculares se ha centralizado y concentrado en «los expertos» que desde la capital del país seleccionan y difunden los lineamientos que habrán de seguir todas las comunidades escolares (Pinto, 2008; Osandón, Caro, Abraham, Magendzo, Abraham, Lavín, González y Cabaluz, 2018; Caro, 2018).

$\mathrm{Al}$ analizar el momento histórico que se ha abierto en Chile, creemos que la racionalidad curricular técnico-instrumental y sus efectos de concreción en las comunidades educativas, son una barrera para el desarrollo de una educación inclusiva, equitativa y democrática (Unesco, 2019); y, por lo tanto, una piedra de tope que impide una real democratización de la sociedad chilena, al 
menos desde el ámbito escolar. En este sentido, comprendemos el currículum desde una conceptualización multidimensional, el cual sobrepasa los límites del currículum prescrito en documentos y lineamientos ministeriales, desde el reconocimiento de las experiencias de los actores que participan del proceso educativo, como sujetos con capacidad de construir currículum y de tener una participación activa en su análisis, generación de propuestas y trabajo (Johnson-Mardones, 2015).

En confluencia con las propuestas pedagógicas de Paulo Freire (1970, 2008), y de variadas teorías y corrientes pedagógicas, cuyo horizonte es una educación democrática que ponga en juego la experiencia, las necesidades, las esperanzas y los conocimientos de los sujetos que son parte de los procesos educativos, asumimos la posibilidad de comprender el currículum desde una perspectiva democrática y vinculada a la realidad: un currículum construido con la participación de sus propios actores, relacionado con la reflexión de la propia práctica y con la forma de organizar los procesos educativos (Galeano, 2001) para la construcción de un conocimiento situado y vinculado a los sentidos y propósitos de la comunidad, para «aprender la democracia, en la propia vivencia de esta» (Freire, 2008, p. 86).

El rol que puede tener el profesorado en la construcción democrática del currículum es sin duda un elemento fundamental. En contraposición a las consecuencias precarizadoras que han tenido las políticas neoliberales que han reducido la capacidad de acción y las potencialidades de los trabajadores de la educación, este es un actor clave para la construcción de proyectos educativos anclados en la realidad (Mejía, 2010). Sin duda, el fortalecimiento y el empoderamiento de un profesorado con mayores niveles de autonomía pedagógica y laboral nos parece una condición sin la cual, la construcción de una propuesta educativa contextualizada con un horizonte educativo democrático se hace imposible.

\subsection{Construcción curricular y currículum de emergencia}

Frente a este panorama histórico, y ante las problemáticas señaladas, la irrupción del COVID-19 a Chile llegó a visibilizar y profundizar aún más las desigualdades estructurales del país. A dos semanas de iniciarse el año escolar, se debieron suspender las clases presenciales sin un plan de acción, orientación y apoyo por parte del Ministerio de Educación (Mineduc). Conviene mencionar que, entrando a una crisis sanitaria que afectaba gravemente a las poblaciones más vulneradas, el Ministerio hizo un llamado a «aprender sin parar,» una propuesta que abogaba por continuar la cobertura curricular 
en las diferentes disciplinas, sin contemplar las posibilidades de acceso para la educación remota o la situación de las comunidades educativas.

El momento de crisis obligó a cada escuela a adaptarse a las condiciones que imponía la pandemia y las condiciones sociales de la comunidad escolar desde sus propias trayectorias, horizontes y posibilidades. Ante la ausencia de apoyo y de la normal imposición curricular del Mineduc, se abrió entonces un momento donde se hizo vital para las instituciones escolares, pensarse a sí mismas, el sentido de la educación y las posibilidades que brindaba un complejo escenario pandémico, económico, sanitario, político y social, un momento que hacía aún más urgente la construcción de propuestas educativas vinculadas a la realidad, conocedoras de los contextos, atentas a las necesidades e intereses de los actores, y promotoras del involucramiento de sus comunidades.

En este contexto, el Mineduc (2020a, 2020b) anunció una «priorización curricular» aprobada por los organismos que sancionan la prescripción curricular nacional y programas de estudio, y pensada para un eventual regreso a la escuela presencial que nunca ocurrió durante el año 2020. Esta priorización curricular centralizada y diseñada para la cobertura curricular consistió en una selección de objetivos de aprendizaje considerados imprescindibles a los que las escuelas deberían abocarse para dar cumplimiento a los aprendizajes esperados de cada nivel y asignatura del currículum nacional. Esta decisión centralizada ocasionó y profundizó una serie de procesos de toma de decisiones curriculares a nivel local y de escuela, surgiendo distintas iniciativas y propuestas de construcción y desarrollo curricular. Estos procesos fueron dispares en su profundidad y duración, dado que la priorización fue definida como una selección de objetivos de aprendizaje, la unidad principal de los instrumentos curriculares chilenos. Algunas de estas experiencias adoptaron denominaciones como: priorización, articulación, nuclearización. Lo común de estos desarrollos fue la toma de decisiones por parte de las comunidades profesionales con mayor o menor participación de las comunidades educativas en general y con mayor o menor consideración de la propuesta del Mineduc.

Una de estas alternativas para denominar los procesos de construcción curricular desde abajo fue el de nuclearización. El concepto fue propuesto por el Colegio de Profesores de Chile quienes ofrecieron un marco conceptual para desarrollar procesos de nuclearización curricular que permitieran un trabajo más contextualizado e integrado a nivel escuela.

Proponemos por ello, un cambio de perspectiva que implica la nuclearización del currículum, que equivale a la resignificación cualitativa de dicho referente 
y a impulsar procesos de síntesis y contextualización de los aprendizajes. Esto puede realizarse a partir de diversas acciones que permitan determinar y/o elaborar los saberes, habilidades y elementos del contexto más relevantes, así como las formas de interpretación más generales para promover una comprensión holística de los campos temáticos abordados. Y, junto a ello, abre la necesidad de reivindicar el rol docente en tanto productor/constructor de currículo y no un simple receptor e implementador de este (Soto, 2003). (Colegio de Profesores de Chile, 2020)

Es importante destacar que la propuesta sugirió distintas formas de nuclearización posibles y presentó ejemplos de desarrollo o construcción curricular enviados por escuelas o docentes para ser compartidos a nivel nacional. Entre las propuestas se presentaban nuclearización por articulación de asignaturas, nuclearización por categorización de saberes, y nuclearización por reelaboración e integración de objetivos de aprendizajes.

Con todo, es necesario decir que el proceso de documentación de estas experiencias de trabajo curricular desarrollado por profesores y comunidades educativas está solo comenzando. Este trabajo pretende ser una contribución en esa línea.

\section{Metodología}

El presente trabajo tiene como objetivo describir el proceso de construcción curricular participativa de las y los trabajadores de la educación de un colegio particular-subvencionado, en el marco de la pandemia de COVID-19. El estudio se enmarca en una escuela financiada por el estado y administrada por una fundación privada sin fines de lucro (subvención compartida) ubicada en la comuna de Peñalolén en la ciudad de Santiago de Chile. La comuna se encuentra ubicada en el sector sur oriente de la ciudad, existiendo como tal desde 1981. Tiene una población aproximada de 253000 habitantes y presenta un índice de vulnerabilidad mayor al de la región metropolitana (Municipalidad de Peñalolén, s.f). El equipo de investigación estuvo compuesto por integrantes del Núcleo de Estudio Currículum, Conocimiento y Experiencia Escolar (NECCEE) de la Universidad de Chile, que incluyeron una trabajadora y un trabajador del establecimiento que participaron del proceso, junto a un investigador asociado. De esta forma, se pretendió documentar y analizar la experiencia vivida por una comunidad escolar desde abril hasta diciembre de 2020.

Esta propuesta fue construida y realizada por 16 trabajadores y trabajadoras de la educación, entre los que se encuentran profesores y profesoras 
de primera infancia, de educación especial, de primaria, de asignaturas específicas como educación física, música e inglés, y por profesionales asistentes como técnicos de aula y terapeutas del lenguaje. Estos mantenían reuniones de coordinación de manera sistemática un día a la semana para comentar, planificar y reflexionar en torno a la propuesta en construcción, además de reuniones específicas por curso y/o por proyectos de trabajo mediante plataformas de videoconferencia. Dentro de las actividades centrales del trabajo colectivo se encontraba la creación de estructuras de trabajo para la organización del grupo y lo relacionado con el ámbito curricular, la planificación de experiencias de aprendizaje en torno a un tema generador, la elaboración de materiales asincrónicos para el desarrollo de las actividades por parte de los estudiantes, y la distribución de tareas para el desarrollo de la propuesta curricular. La participación de estudiantes del nivel preescolar hasta tercero de primaria, correspondientes a la coordinación de primer ciclo llamada Kimün, se desarrolló a través de la realización de actividades educativas asincrónicas por medio de las redes sociales de Facebook y WhatsApp y de la participación en experiencias sincrónicas de diálogo realizadas en la plataforma Zoom que tenían como objetivo realizar actividades colectivas, comentar y sugerir temas de interés, y elegir tópicos a trabajar en el tema generador.

Para la documentación de la experiencia, se definió un diseño de investigación documental (Kridel, 2010; Scott, 2006; McCulloch, 2004) a partir de datos secundarios que se han desarrollado a lo largo de la experiencia, los cuales fueron producidos de forma previa al proceso de documentación (Vieytes, 2004). Se optó por este tipo de investigación debido a su potencial para relacionar asuntos y documentos personales, con temas públicos, educativos, culturales o sociales, así como también, por establecer nexos entre asuntos desarrollados en el pasado con el presente, a partir de la investigación de diferentes tipos de documentos (McCulloch, 2004). De esta manera, la investigación documental en Estudios Curriculares va «más allá que simplemente usar documentos como fuente de datos» siendo su intención también «el informar mediante la construcción de documentos» para «revelar eventos o situaciones auténticas en forma autentica y realista" siendo cierta "una forma de autentificación de eventos, situaciones, o formas de vida» (Kridel, 2010, p. 299).

Dentro de los documentos utilizados, se utilizaron tres tipos: textos de trabajo de grupal (planificaciones, reflexiones, otros), actas de registro de las reuniones colectivas de coordinación del ciclo y las entradas realizadas en el cuaderno de campo de un trabajador y una trabajadora del establecimiento. 
Se utilizó como técnica el análisis de contenido (Flick, 2004, 2015) y como base la guía que propone Bardin (1996) acerca del uso de dicha técnica, y se realizaron modificaciones según el objetivo y contexto de este trabajo. Como se trata de un análisis con datos secundarios que buscan una documentación general de la experiencia, se utilizan los documentos señalados anteriormente como unidades de registro, mediante la categorización en base a temas como núcleos de sentido (Freire, 1970, 2008). El corpus seńalado cumple con criterios de representatividad de la muestra en relación con el universo de documentos que fueron producidos a lo largo de la experiencia, y a la pertinencia en cuanto a la conexión que guardan con el objetivo de documentar la experiencia curricular en cuestión. Así, los documentos seleccionados son representativos de las instancias de reunión entre los y las participantes, de las actividades generadas a partir de dichas reuniones y de materiales de organización y planificación del equipo de trabajo.

En relación con lo anterior, se realiza un primer análisis de los datos de modo de hacer una lectura inicial de los datos existentes y los géneros de los documentos que fueron analizados (visuales y textuales) y se levantaron temas emergentes para el análisis de los documentos bajo la pregunta ¿Qué elementos caracterizan la propuesta curricular? Posteriormente, se profundizó la categorización temática para refinar los temas emergentes en la relación entre sí y con otros de modo de agrupar la información en categorías temáticas más generales. En última instancia, se desarrolla una fase de interpretación e inferencia a partir de los datos categorizados (Arbeláez y Onrubia, 2014; Bardin,1996; Delgado y Gutiérrez, 1995; Flick, 2004).

Desde el punto de vista ético, la difusión de las actividades de estudiantes y sus familias en las diversas instancias de esta experiencia contaron con su consentimiento en cuanto a los efectos de la utilización de fotografías y videos, a pesar de ser publicadas en una cuenta pública de Facebook. En el caso que la familia no quisiera que las actividades fueran publicadas en esa vía, se entregaron opciones para resguardar la identidad y la imagen de los niños y las niñas.

\section{Resultados}

\subsection{Describiendo la experiencia}

La experiencia se desarrolló en dos etapas, las que presentamos a continuación, junto con las formas de organización presente en cada una de ellas. La primera la caracterizamos como de exploración, marcada por un momento de crisis de funcionamiento del sistema escolar, incluyendo la reflexión colectiva a partir 
de preguntas y de la identificación de barreras cuya superación permitiera a todo el estudiantado sentirse parte, aprender y participar de la experiencia escolar en el contexto de emergencia, y por un momento de acción, donde se realizaron los primeros intentos de una nueva propuesta curricular. La segunda etapa la denominamos de consolidación debido a que la propuesta se robusteció, generando una dinámica de construcción curricular, de acción y reflexión permanente, relacionada al desarrollo de propuestas pedagógicas para abordar problemáticas e intereses de la comunidad educativa.

\section{Etapa de exploración}

En primer lugar, se presentarán las problemáticas iniciales que se abordaron en el origen de la propuesta curricular. Estos temas fueron utilizados como base para el desarrollo de la reflexión docente, la cual se llevó a cabo a través de reuniones a distancia. El cuestionamiento constante, así como el análisis de las problemáticas planteadas es abordado desde las emociones y sentidos de los y las trabajadoras de la educación, considerando el contexto actual y las preocupaciones e intereses propios de la comunidad educativa. Algunas preguntas que orientaron constantemente el trabajo colaborativo y constituyeron un desde para pensar y reflexionar en torno a las prácticas educativas del establecimiento, fueron las siguientes:

- ¿Qué sentido tiene la educación para nosotros y nosotras?

- ¿Qué sentido le atribuimos a aprender? ¿Para qué aprendemos?

- ¿Qué condiciones materiales y emocionales tienen las y los trabajadores, y las familias de nuestra comunidad educativa?

Las respuestas emergidas orientaron el trabajo colectivo y su organización. Primero, se reconocieron dificultades emocionales y personales que se viven en el contexto de confinamiento como las labores propias del hogar (las cuales por lo general están a cargo de las mujeres), el hacinamiento y las dificultades económicas que viven las familias de la comunidad escolar; y las dificultades iniciales de acceso a internet para conectarse a clases de tipo sincrónicas. En segundo lugar, existe un consenso entre las y los trabajadores en torno a la desconexión que se da frecuentemente entre la escuela y la realidad, la que pareciera ser inaceptable dado el contexto de emergencia, el cual necesita de una educación pertinente que permita brindar oportunidades de aprendizaje y participación para todos y todas, con un foco transversal en el ámbito emocional. En esta línea, se plantea una crítica a la parcelación del 
conocimiento por asignatura y, finalmente, se destaca el sentido comunitario de la educación y de la escuela, que, ante el confinamiento y la distancia física, requiere de la generación de espacios donde todos y todas se sientan parte.

A partir de estas reflexiones, se decidió producir materiales audiovisuales con actividades educativas, las cuales eran publicadas en Facebook. La elección de esta plataforma fue debido a que en Chile su acceso era gratuito en el contexto de pandemia y que la mayoría de las familias eran parte de esta red social y podían acceder a esta de forma asincrónica. En ese momento se hizo relevante enfocar las actividades en la contención emocional, en el autocuidado, en las distintas formas de aprender, así como también, en los materiales y recursos con los que contaban los y las estudiantes en sus hogares.

Como se presenta en la Figura 1, la organización curricular de este momento se generó a través de seis «centros de aprendizaje», desde los cuales se buscaba desafiar e intencionar aprendizajes desde diferentes dimensiones. Cada uno de estos Centros era conformado por duplas o tríos de trabajo que producían y publicaban actividades con regularidad, y buscaban abordar, desde los objetivos de diferentes disciplinas, un tema cotidiano del quehacer y el vivir bajo la pandemia y en contexto de confinamiento.

Figura 1. Organización curricular inicial marzo-mayo 2020

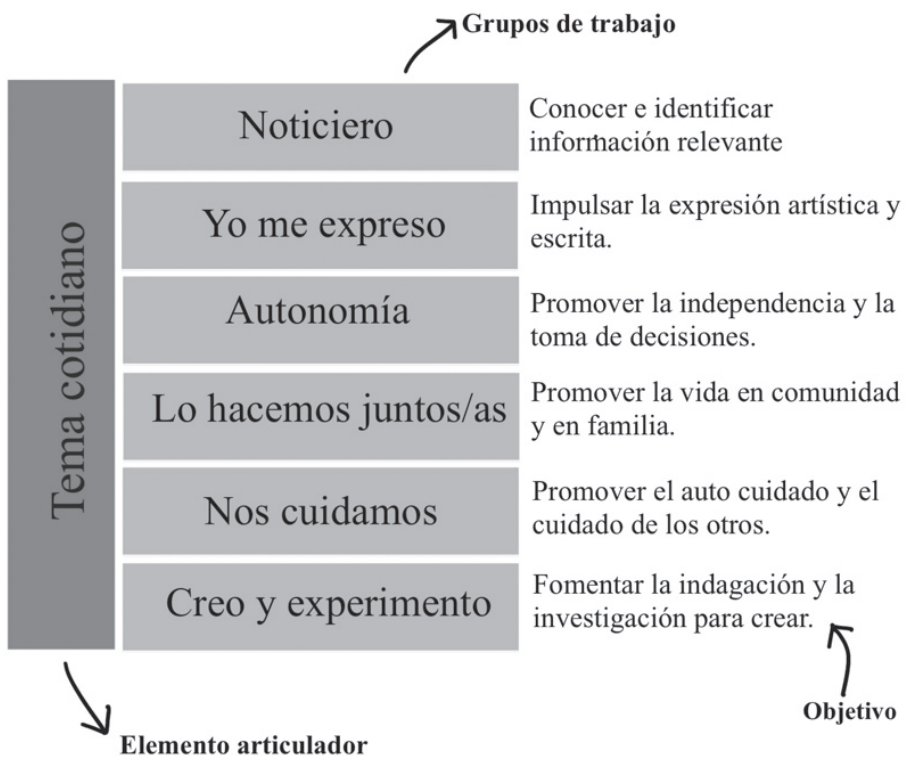




\section{Etapa de consolidación}

Con el desarrollo de la propuesta curricular del primer momento, se fueron vislumbrando algunas problemáticas como la falta de vinculación entre los temas trabajados por cada centro de aprendizaje, la desconexión entre las y los trabajadores de distintos grupos y la necesidad de desplazarse de objetivos individuales o grupales aislados hacia el reforzamiento del sentido comunitario de la propuesta educativa. Desde estas reflexiones se dio inicio a la que hemos denominado la segunda etapa en el mes de junio de 2020, en la cual se fue consolidando la propuesta a través de la generación de proyectos, de temas generadores y de la nuclearización de objetivos. Consecuentemente, se decidió pasar de los seis «centros de aprendizaje» a cuatro «proyectos» conformados por cuatro grupos de trabajo. El proyecto debía sostenerse en el tiempo y constituirse como un eje articulador, un lugar desde el cual se difundieran materiales audiovisuales con actividades de aprendizaje relacionadas al tema generador y a los núcleos de aprendizaje durante toda una semana.

Además, basándose en la propuesta de Paulo Freire (1970), se transitó del concepto de «tema cotidiano» al de «tema generador», es decir, hacia el tratamiento de una problemática o un interés que emerge desde la propia comunidad. A partir de un tema o una problemática se definía una pregunta y varios ejes temáticos que conformaban la totalidad del tema generador. Por otra parte, para facilitar la planificación de actividades, se generaron «subejes» con temas más específicos. De esta forma, se logró articular la propuesta pedagógica de cada proyecto en el abordaje de uno o más ejes y subejes del tema generador, desarrollando actividades particulares, pero enmarcadas por una unidad temática mayor vinculada a la realidad de las y los estudiantes. En la definición de los temas generadores se buscó, de manera progresiva, generar espacios de diálogo sincrónico para involucrar a las y los estudiantes en la toma de decisiones.

Por último, se buscó integrar los objetivos de aprendizaje establecidos en las bases curriculares del Ministerio de Educación (Mineduc, 2012) mediante una «nuclearización», desde la cual se combinaron y asimilaron objetivos de diferentes disciplinas en una propuesta en la cual se establecieron cinco grandes núcleos que a su vez estaban conformados por otros núcleos más pequeños. Esto permitió una mayor integración de los objetivos de las diferentes disciplinas. 
Figura 2. Propuesta de nuclearización

\begin{tabular}{|c|c|c|}
\hline CONOCIMIENTO & $\begin{array}{l}\text { Valoración del rol de las } \\
\text { naturaleza }\end{array}$ & Pensamiento temporal \\
\hline $\begin{array}{l}\text { DEL MUNDO QUE } \\
\text { ME RODEA }\end{array}$ & $\begin{array}{l}\text { Flora, fauna y paisajes } \\
\text { Investigación }\end{array}$ & $\begin{array}{l}\text { Comprensión critica de la } \\
\text { sociedad en la que } \\
\text { vivimos. }\end{array}$ \\
\hline \multirow{2}{*}{ ESCRITURA } & Motricidad fina & \multirow[t]{2}{*}{ Escritura creativa } \\
\hline & Motricidad gruesa & \\
\hline \multirow{4}{*}{ LECTURA } & Escucha de historias & Comprensión lectura \\
\hline & \multirow{2}{*}{$\begin{array}{l}\text { Lectura de palabras de } \\
\text { forma independiente }\end{array}$} & Fluidez \\
\hline & & \multirow{2}{*}{$\begin{array}{l}\text { Lectura de textos } \\
\text { diversos (en estructura y } \\
\text { función) }\end{array}$} \\
\hline & Goce lector & \\
\hline \multirow{3}{*}{$\begin{array}{l}\text { PENSAMIENTO } \\
\text { LÓGICO } \\
\text { MATEMÁTICO }\end{array}$} & Numeraciốn & \multirow{2}{*}{$\begin{array}{l}\text { Comparar, clasificar y } \\
\text { patrones }\end{array}$} \\
\hline & Geometria & \\
\hline & Operaciones & Resolucióa de problemas \\
\hline \multirow{3}{*}{$\begin{array}{l}\text { EXPRESIÓN y } \\
\text { COMUNIDAD }\end{array}$} & Desarrollo de ideas & \multirow[t]{2}{*}{$\begin{array}{l}\text { Expresión de emociones y } \\
\text { opiniones }\end{array}$} \\
\hline & Expresión oral & \\
\hline & Respeto por los demás & $\begin{array}{l}\text { Creación de expresiones } \\
\text { artisticas }\end{array}$ \\
\hline
\end{tabular}

Fuente: Elaboración propia sobre la base de la propuesta curricular de la escuela.

Figura 3. «Proyectos» de la Organización curricular (de junio a diciembre 2020)

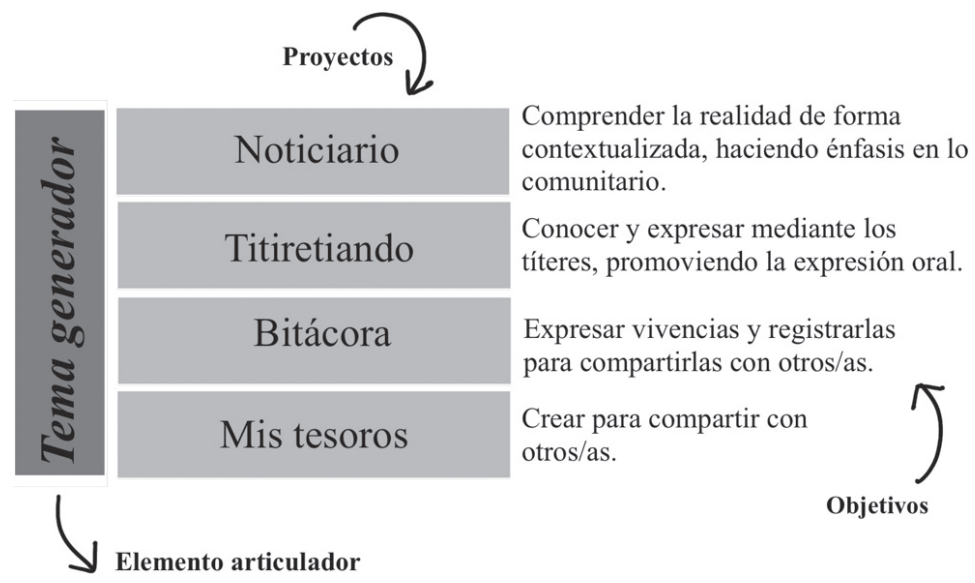

Fuente: Elaboración propia sobre la base de la propuesta curricular de la escuela. 


\subsection{Principios e implicancias de la experiencia}

A pesar de ser una experiencia incipiente, nos parece relevante poder enfatizar los resultados preliminares de esta propuesta de modo de avanzar hacia su sistematización y documentación y así aportar a la construcción de conocimiento en esta área, a las mejoras dentro de la propia institución, y a otros centros educativos en la búsqueda de alternativas al currículum prescrito. Destacamos cuatro principios orientadores que nos parece representan la esencia de la experiencia y los cuales consideramos claves en la construcción de una propuesta curricular acorde a una educación equitativa y de calidad para todos y todas.

1. La organización del currículum a partir de temas generadores. Dentro de los diálogos y reflexiones por parte del profesorado, el tema del sentido de la educación ha emergido como un elemento crítico. Al respecto, los temas generadores nos entregan la posibilidad no solo de acercar la realidad a la escuela sino también de articular las distintas experiencias de aprendizaje en torno a un problema situado, relevante y común. En ese sentido, urge abandonar el sentido de la educación desde una concepción bancaria para avanzar hacia una educación dialógica, lo que implica una mayor integración de los procesos de diseño y desarrollo curricular que considere lo local en ambos procesos.

2. La nuclearización de objetivos de aprendizaje. Comprender el aprendizaje de forma holística resulta fundamental a la hora de generar un currículum significativo y anclado con la realidad. Lo anterior, implica reconocer el aprendizaje como una experiencia que no solo se genera en la escuela sino en todos los lugares de la vida, que se realiza de manera constante, que no se pierde y que se construye directamente con las experiencias de cada uno. En ese sentido, las disciplinas escolares pueden organizarse de manera distinta y ser articuladas bajo un fin compartido avanzando hacia la superación de la fragmentación y saturación del currículum por disciplinas y objetivos de aprendizaje.

3. El trabajo multigrado y la diversificación de actividades de aprendizaje. La división de la experiencia escolar en torno a la edad del estudiantado impide una valoración de la diversidad de formas de aprender y participar, esperando resultados y características similares en grupos de estudiantes de una misma edad. La propuesta presentada promueve objetivos y actividades amplias y flexibles con temáticas diversas de modo de adaptarse a todos y todas las estudiantes, independientemente de su edad, nacionalidad o características personales. Desde ahí, se 
reconoce la diversidad como un valor inherente a los grupos humanos por lo que un currículum flexible que respete las trayectorias de vida y las potencialidades de quienes participan del proceso educativo permite poner en valor y enriquecer las experiencias educativas.

4. La capacidad de los y las trabajadoras de la educación como agentes legítimos de construcción curricular. Parte de la democratización del conocimiento y por ende del propio currículum, es valorar y rescatar la participación de todos los actores en su construcción. Desde allí, reconocemos la importancia de los y las maestras como generadores de currículum y no de meros implementadores. Lo anterior, se ve favorecido gracias al conocimiento que se tiene de los y las estudiantes y de su contexto, las experiencias de cada actor como sujeto político, sumado a la capacidad de autoformación constante para el desarrollo de esta propuesta.

\section{Discusión}

Con la irrupción del COVID-19 en Chile, se agudizaron las problemáticas sociales que habían llevado al país a un momento de quiebre de la institucionalidad política, al tiempo que se interrumpió el funcionamiento típico del sistema escolar. Las dificultades sanitarias, socioeconómicas y el traslado de los procesos escolares al ámbito familiar que planteaba el escenario de la pandemia, hizo aún más urgente el desarrollo de una propuesta educativa pertinente y vinculada a las experiencias de vida de las comunidades. Así, pensar la escolarización en pandemia como una zona de indeterminación, una escolaridad en suspensión, una escuela ausente, es posible pensar también las posibilidades que esta situación ha conllevado. Estas posibilidades no surgen solo de la reflexión intelectual sino de un conjunto de experiencias vividas en dicho contexto. En este sentido, Boaventura de Sousa Santos nos dice que

L[1]a pandemia y la cuarentena revelan que hay alternativas posibles, que las sociedades se adaptan a nuevas formas de vida cuando es necesario y se trata del bien común. Esta situación es propicia para pensar en alternativas a las formas de vivir, producir, consumir y convivir en los primeros ańos del siglo XXI. (Santos, 2020, p. 79)

En este sentido, reconociendo esta experiencia como una vivencia situada en el contexto chileno, nos parece necesario identificar barreras del sistema educativo y de la sociedad que impiden avanzar hacia una sociedad más justa y que dificultan el camino de la educación inclusiva. Muchas de ellas, 
son cuestionadas por la comunidad estudiada y son parte fundamental del sistema educativo: el currículum y el rol del profesorado.

Desde el ámbito del currículum, una primera barrera dice relación con una tradición curricular marcada por una racionalidad técnica y por el centralismo curricular. Este no es solo un problema de selección y organización de contenidos sino también un problema político en tanto se excluye la participación en la toma de decisiones curriculares a las propias comunidades, se imponen contenidos culturales propios de la cultura dominante y en el caso del Chile actual, se orientan los procesos pedagógicos a la satisfacción de las necesidades del mercado (Pinto, 2008; Osandón, et al., 2018; Caro, 2018). Desde el organismo encargado de la construcción del currículum se exige el cumplimiento de una gran cantidad de contenidos y habilidades que generan una saturación de objetivos que muchas veces son inabarcables por el profesorado. Por último, el sentido común presente en la sociedad, que asocia las actividades escolares con una tradición bancaria (Freire, 1970) también representaría una barrera para poder romper las lógicas tradicionales excluyentes.

Concretamente, lo anterior se constituye como un obstáculo para pensar un currículum flexible y adaptable a la diversidad. En ese sentido, la propia Unesco (2019) reconoce la necesidad de transformar el currículum, abriendo sus horizontes e implicaciones más allá de los contenidos disciplinares, para avanzar hacia conocimientos, habilidades y actitudes para aprender a vivir en comunidad y en diversidad. Así, releva la importancia de la participación de los actores sociales vinculados a la educación para el diseño de marcos legales y políticas educativas. Desde ahí, la propuesta de los temas generadores permitió a las y los trabajadores de la educación participar del proceso de construcción curricular abordando temas y problemáticas de la comunidad escolar. En ella, existe un reconocimiento y una valoración de la diversidad del alumnado y sus familias y una orientación clara hacia la producción de saberes de vida a partir de la cotidianidad de los niños y nińas. La participación de diversos actores de la comunidad propicia una cultura democrática y promoviendo un currículum generado desde el territorio.

De estos planteamientos se desprende que el profesorado tiene un rol clave en la construcción de una propuesta educativa pertinente y democrática, como se muestra en el caso de la experiencia presentada. La literatura revisada plantea que en las últimas décadas las políticas neoliberales han impulsado un proceso de desprofesionalización, despedagogización y de precarización de la labor docente a nivel latinoamericano (Mejía, 2010). Esto se contrapone con las investigaciones que sitúan al profesorado como agentes de cambio para la justicia social, relevando los sentidos personales para promover condiciones de justicia, la autonomía, las 
competencias propias para diseñar entornos inclusivos y por cierto la capacidad de reflexión para mirar su práctica de manera constante (Pantic, 2015).

Desde la experiencia descrita en este trabajo, es posible observar algunos de estos elementos como la capacidad de autonomía al vincular la acción pedagógica con el sentido de educar para todos, el trabajo colaborativo para desarrollar la propuesta y la reflexión por los sentidos profundos de la educación desde una perspectiva situada en su realidad y momento histórico, dan cuenta del potencial transformador que puede tener una comunidad educativa que se piensa, se proyecta y aprende de su propia experiencia como territorio de saberes. Si bien, desde el trabajo colaborativo de trabajadores y trabajadoras de la educación, y de una experiencia de integración curricular no se resuelven inmediatamente las desigualdades estructurales, entendemos que estos son elementos necesarios para una educación verdaderamente inclusiva y que pueden ser aportes para avanzar en procesos de democratización de la sociedad.

A modo de cierre, concluimos que, a pesar de las limitaciones curriculares, de precarización del ejercicio docente, y las condiciones adversas que planteaba el contexto de pandemia, esta experiencia da cuenta del potencial educativo del que son capaces las y los trabajadores de la educación en condiciones de mayor autonomía profesional y de una praxis vinculada a la realidad de las comunidades educativas.

\section{Conclusiones}

La experiencia documentada nos ha abierto un camino de posibilidades y horizontes tanto para la propia escuela como para otras comunidades. A saber, la progresiva participación de todos los actores de la comunidad en la construcción curricular a través de la definición de los temas generadores; una mayor apropiación del sentido de la educación en perspectiva transformadora; el desarrollo de múltiples instancias de sistematización, documentación y producción de conocimiento desde esta experiencia; la multiplicación de instancias educativas multigrado; la proyección de políticas curriculares que abran espacio a este tipo de propuestas educativas; y finalmente, el desafío de traspasar la experiencia desarrollada a la modalidad presencial. Por último, la propuesta nos sitúa desde una concepción del currículum como experiencia vivida y, por ende, necesaria de documentar y sistematizar. Lo anterior resulta fundamental en Chile, que actualmente vive un proceso de transformación político, social y económico. La educación, por tanto, también debiese transformarse y construirse desde abajo a partir de la reflexión colectiva para pensar $\mathrm{y}$ vivir en una sociedad más equitativa, democrática y justa. 


\section{REFERENCIAS BIBLIOGRÁFICAS}

Arbeláez, M. y Onrubia, J. (2014). Análisis bibliométrico y de contenido. Dos metodologías complementarias para el análisis de la revista colombiana. Educación y Cultura. Revista de Investigaciones UCM, 14(23), 14-31. https://doi.org/10.22383/ri.v14i1.5

Agamben, G. (2003). Infancia e historia. Buenos Aires: Adriana Hidalgo.

Assael, J., Cornejo, R., Albornoz, N., Echeberriegaray, G., Ligueño, S. y Palacios, D. (2015). La crisis del modelo educativo mercantil chileno: un complejo escenario. Curriculo sem Fronterias, 15(2), 334-345. Recuperado de https://www.curriculosemfronteiras.org/vol15iss2articles/assael_etal.pdf

Bardin, L. (1996). Análisis de contenido. Madrid: Akal.

Caro, M. (2018). Red de escuelas con sello experimental: hacia un proyecto de escuela pública para una vida digna, justa, democrática y socialmente protagónica. Revista Docencia, (63), 63-69. Recuperado de http://revistadocencia.cl/web/images/ediciones/Docencia_63.pdf

Caro, M. y Aguilar, M. (2018). Desarrollo del curriculum en las aulas: perspectivas desde el profesorado. En A. Arratia, y L. Osandón Marivil (eds.). Politicas para el desarrollo del curriculum: Propuestas y tensiones. Santiago: MINEDUC-UNESCO.

Colegio de Profesores de Chile. (2020). Orientaciones para el trabajo pedagógico a partir del plan educativo de emergencia para enfrentar la crisis sanitaria del colegio de profesoras y profesores de chile. Recuperado de http://revistadocencia.cl/web/index.php/orientaciones

Connelly, M. y Clandinin, J. (1988). Teachers as Curriculum Planners: Narratives of Experience. New York, NY: Teachers College Press.

Cornejo, R. (2018). Políticas y reformas escolares: el experimento educativo chileno y su evolución. En C. Ruiz, L. Reyes, y J.F. Herrera (eds.), Privatización de lo público en el sistema escolar. Chile y la agenda global de educación (pp. 237-263). Santiago: LOM Editores.

Clandinin, J. y Connelly, M. (1992). Teacher as currículum maker. En P. W. Jackson (ed.), Handbook of research on curriculum: a project of the American Educational Research Association (pp. 363-401). New York: MacMillan

Delgado, J. y Gutiérrez, J. (1995). Métodos y técnicas cualitativas de investigación en ciencias sociales. Madrid: Editorial SINTESIS S.A.

Díaz-Barriga, A. (2020) La escuela ausente, la necesidad de replantear su significado. En Aguilar et al. Educación y Pandemia. Una visión académica. México: Instituto de Investigaciones sobre la Universidad y la Educación, UNAM. 
Espinoza, O., Guzmán, M.A. y Riquelme, S. (2018). Currículum nacional y oportunidades de desarrollo curricular local: Proyecto curricular, programas de studio y plan de formación ciudadana. En A. Arratia y L. Osandón (eds.). Politicas para el desarrollo del curriculum: Propuestas y tensiones. Santiago: Mineduc-Unesco.

Erazo, M. S. (2011). Innovación de las prácticas de planificación curricular en la escuela y en el liceo: Una estrategia para la apropiación, contextualización y complementación de los planes y programas propuestos por el Mineduc. Revista Pensamiento Educativo, 29(12), pp. 245-275.

Ferrada, D. (2012). Construyendo escuela, compartiendo esperanzas. La experiencia del proyecto Enlazando Mundos. Concepción, Chile: Ril EditoresEditorial UCSC.

Ferrada, D. (2001). "Comunidades de entendimiento»: Una propuesta educativa desde la perspectiva crítica comunicativa del currículum. Revista Pensamiento Educativo, 29(1), 297-317.

Flick, U. (2004). Introducción a la investigación cualitativa. Madrid: Morata.

Flick, U. (2015). El diseño de investigación cualitativa. Madrid: Morata.

Foro por el Derecho a la Educación Pública (2019). Informe a la luz de la situación de la educación en Chile al 2019. Recuperado de http://derechoeducacionpublica.cl/wp-content/uploads/2019/07/FODEP-2019-Informe-Luz-dela-Situación-de-la-Educación-en-Chile.pdf

Freire, P. (1970). Pedagogía del oprimido. Buenos Aires: Siglo Veintiuno Editores.

Freire, P. (2008). La educación como práctica de la libertad. Buenos Aires: Siglo Veintiuno Editores.

Galeano, J. (2001). Construcción curricular y cotidianidad escolar. Uni-Pluril versidad, 1(1), 39-48. https://revistas.udea.edu.co/index.php/unip/article/ view/12377

Gentili, P. (2001). La exclusión y la escuela: el apartheid educativo como política de ocultamiento. Revista Docencia, 15(1), 4-11. Recuperado de http:// revistadocencia.cl/ revist37/web/images/ediciones/docencia_15.pdf

Glatthorn, A., Boschee, F., Whitehead, B. y Boschee, B. (2019). Curriculum leadership: Development and implementation. Thousand Oaks, CA: SAGE.

Giroux, H. (1990). Los profesores como intelectuales. Buenos Aires: Paidos.

Grez, S. y Foro por la Asamblea Consituyente. (2015). Asamblea constituyente: la alternativa democrática para Chile. Santiago: Editorial América en movimiento.

Grundy, S. (1987). Producto o praxis del curriculum. Madrid: Morata. 
Guzmán, M. (2001). Emergentes: Una aproximación teórica para comprender el significado innovador de la producción curricular intra-aula. Revista Pensamiento Educativo, 29(1), 319-340.

Gysling, J. (1992). Profesores y currículum: Un estudio cualitativo. Santiago: CIDE. Hlebowitsh, P. (2009). Navigating the Normative. Curriculum Inquiry, 39(1), 15-26. https://doi.org/10.1111/j.1467-873X.2008.01434.x

Johnson-Mardones, D. (2015). Understading Curriculum as Phenomenon, Field and Design: A multidimensional conceptualization. Journal of International Dialogues in Education, 2(2), 1-9.

Kridel, C. (2010). Encyclopedia of Curriculum Studies Encyclopedia of curriculum studies. Thousand Oaks, CA: SAGE Publications, Inc. https://doi. org/10.4135/9781412958806

Lozano G., C. (2018). El consejo escolar del Instituto Nacional (JoséMiguel Carrera) desde una perspectiva de deliberación curricular. Estudios Pedagógicos, 42(2), 207-226. https://doi.org/10.4067/S0718-07052016000200012

McCulloch, G. (2004). Documentary research in education, history and the social sciences. Londres: Routledge Falmer. https://doi.org/10.4324/9780203464588

Mejía, M. (2010). Las pedagogías críticas en tiempos de capitalismo cognitivo. Cartografiando las resistencias en educación. Planetapaz. Recuperado de http://www.cepalforja.org/sistem/documentos/pedagogias_criticas.pdf

Mineduc. (2020a). Fundamentación priorización curricular COVID-19. Santiago: Mineduc.

Mineduc. (2020b). Priorización curricular COVID-19. Santiago: Mineduc.

Mineduc. (2012). Bases Curriculares: Educación Básica. Santiago: Mineduc.

Municipalidad de Peñalolén. (s.f). Resumen Ejecutivo Diagnóstico Comunal. PLADECO 2013-2016. Ilustre Municipalidad de Peñalolen. Recuperado de https://www.penalolen.cl/wp-content/uploads/2016/10/RESUMENEJECUTIVO-DIAGNOSTICO-PENALOLEN.pdf

Null, W. (2011). Curriculum: From theory to practice. Lanham, MD: Rowman \& Littlefield.

Pantic, N. (2015). A model for study of teacher agency for social justice. Teachers and Teaching: Theory and Practice, 21(6), 759-778. https://doi.org/10.108 0/13540602.2015.1044332

OCDE (2004). Revisión de Politicas Nacionales de Educación: Chile. Recuperado de https://www.oecd-ilibrary.org/education/revision-de-politicas-nacionalesde-educacion-chile_9789264021020-es

Osandón, L., Caro, M., Abraham, M., Magendzo, A., Abraham, M., Lavín, S., González, S. y Cabaluz, J. (2018). Estado, mercado y currículum escolar. 
La experiencia chilena (1964-2018). Santiago: Oficina Internacional de Educación de la Unesco. Recuperado de http://observatoriodocente.cl/ index.php?page =view_recursos\&langSite $=e s \& i d=371$

Pinto, R. (2008). El curriculo critico: Una pedagogía transformativa para la educación latinoamericana. Santiago: Ediciones Universidad Católica de Chile.

Priestley, M., Edwards, R., Priestley, A. y Miller, K. (2012). Teacher Agency in Curriculum Making: Agents of Change and Spaces for Manoeuvre. Curriculum Inquiry, 42(2), 191-214.https://doi.org/10.1111/j.1467-873X.2012.00588.x

Rivera, A. (2009). Código curricular y práctica docente, una relación categorial vigente de analizar desde el contexto de aula. REXE, Revista de Estudios y Experiencias en Educación, 15(8), 51-70.

Sánchez, R., y Sobarzo, M. (2010). El modelo... ¿o el desafío de ser modelo? En I. Cassigoli y M. Sobarzo, Biopoliticas del Sur (pp. 411-419). Santiago: Editorial Arcis

Santos, B. de S. (2020). La cruel pedagogía del virus (Trad. P. Vasile). Buenos Aires: Clacso. https://doi.org/10.2307/j.ctv1gm01nn

Schön, D. (1982). El profesional reflexivo. Cómo piensan los profesionales cuando actúan. Buenos Aires: Paidós.

Scott, J. P. (ed.). (2006). Documentary research. Thousand Oaks, CA: Sage.

Silva, M. (2017). Reforma curricular: regulación y codificación. Madrid: Editorial Académica Española.

Silva, M. (2002). La práctica del discurso curricular. Santiago: Ediciones Cerro Manquehue.

Silva, M. (2001). La Innovación curricular en el contexto de la cultura escolar. Esbozo preliminar. Revista Pensamiento Educativo, 29(1) 231-244.

Stenhouse, L. (1987). La investigación como base de la enseñanza. Madrid: Morata.

Tomasevski, K. (2004). Indicadores del derecho a la educación. Revista Iidh, (40) 341-388. Recuperado de https://revistas-colaboracion.juridicas.unam.mx/ index.php/rev-instituto-interamericano-dh/article/view/8220/7368

Unesco (2017a). Educación en emergencias y crisis. Santiago: Unesco.

Unesco (2019). Foro Internacional sobre Inclusión y Equidad en la Educación. Todas $y$ todos los estudiantes cuentan. Recuperado de https://en.unesco.org/sites/ default/files/2019-forum-inclusion-concept-note-es.pdf

Vieytes, R. (2004) Metodología de la investigación en organizaciones, mercado y sociedad. Epistemología y técnicas. Buenos Aires: Editorial de las Ciencias. 